\title{
Sciendo
}

10.2478/topling-2019-0009

\section{Aspectual coding asymmetries: Predicting aspectual verb lengths by the effects frequency and information content}

\author{
Michael Richter* Giuseppe G. A. Celano** \\ Leipzig University, Germany \\ Natural Language Processing Group
}

\begin{abstract}
The topic of this paper is the interaction of aspectual verb coding, information content and lengths of verbs, as generally stated in Shannon's source coding theorem on the interaction between the coding and length of a message. We hypothesize that, based on this interaction, lengths of aspectual verb forms can be predicted from both their aspectual coding and their information. The point of departure is the assumption that each verb has a default aspectual value and that this value can be estimated based on frequency - which has, according to Zipf's law, a negative correlation with length. Employing a linear mixed-effects model fitted with a random effect for LEMMA, effects of the predictors' DEFAULT i.e. the default aspect value of verbs, the Zipfian predictor FREQUENCY and the entropy-based predictor AVERAGE INFORMATION CONTENT - are compared with average aspectual verb form lengths. Data resources are 18 UD treebanks. Significantly differing impacts of the predictors on verb lengths across our test set of languages have come to light and, in addition, the hypothesis of coding asymmetry does not turn out to be true for all languages in focus.
\end{abstract}

\section{Key words}

aspectual coding asymmetries of verbs, mixed-effects model, information content, frequency, interaction

\section{Introduction}

The present study presents the results of research investigating changes in the lengths of word forms in 18 UD treebanks (Nivre et al., 2017), the research question being whether there is an interaction between aspectual coding of verbs, their information and their lengths.

The aim of the study and choice of the two information features are informed by Shannon's source coding theorem (Shannon and Weaver, 1948) on the interaction of information, coding and length of signs within binary alphabets. We formulate the following research questions: (i) is Shannon's theorem valid for n-ary alphabets and thus for natural languages; and (ii) does the length of aspect-marked verbs interact with their information content and coding?

A previous pilot study on Russian (Celano et al., 2018) revealed that the information of aspectual coded verbs, and also their coding, are strong predictors of verb lengths. The research question in general

\footnotetext{
* Corresponding author: Michael Richter, Leipzig University, Faculty of Mathematics and Computer Science, Natural Language Processing Group, Augustusplatz 10, 04109 Leipzig, Germany. E-mail: richter@informatik.unileipzig.de.

** Giuseppe G. A. Celano participates as an author in that the present article relies on the version which was submitted to, but not accepted by, the UD Workshop 2018 (https://universaldependencies.org/udw18//) as "Celano Giuseppe, Michael Richter, and Rebecca Voll, A mixed-effects model for the imperfective-perfective coding asymmetry with a random effect for lemma" available at https:/github.com/gcelano/udw18.
} 
is influenced by the relationship between length of messages (signs), entropy and frequency as stated by Shannon and Weaver (1948). Shannon's coding theorem states that there is an optimal, informative length for signs. From this theorem it follows that default forms are shorter and thus less informative than non-default forms. The research question in particular is informed by the assumption of a coding asymmetry in natural languages (e.g. Greenberg, 1966; Haspelmath, 2008; Croft, 2012): default aspectcoded verbs are more frequent and shorter than non-default-coded verbs. The former can be said to be zero-coded, while the latter are over-coded. This follows from Zipf's law that "the magnitude of words tends, on the whole, to stand in an inverse (not necessarily proportionate) relationship to the number of occurrences" (Zipf, 1936). It has been argued that Zipf's law applies to a great number and variety of languages (Bentz and Ferreri Cancho, 2016).

Zipf's finding can be interpreted in an information theory framework: from the number of occurrences - that is, absolute frequencies - one can get relative frequencies, i.e. probabilities of the Shannon information content (Shannon and Weaver, 1948) from which information values can be estimated. The Shannon information content is defined as $-\log _{2}(p(u))$, where $u$ is a message. From this it follows that the less frequent signs are (i.e. having lower probability), (i) the longer and (ii) the more informative they will be compared with higher-frequency signs. Zipf (1949) captures this correspondence with the principle of least effort: given two competing forms, it would be uneconomical to use the longer form if it were not also more informative.

In terms of surprisal theory (Hale, 2001; Levy and Jaeger, 2007; Levy, 2008; Levy, 2013), surprisal is equivalent to information and proportional to the difficulty of processing a message. Given this, the hypothesis and research question of this paper follow immediately: because of the interaction between coding, information and length, length can be predicted by information.

What are coding asymmetries in natural languages, and what is meant by the default/non-default dichotomy? Coding asymmetries - namely zero coding vs. overt coding - have been a highly relevant topic in the study of language since Greenberg (1963). Terminologically they are captured as the opposition of markedness to unmarkedness, and thus as "markedness asymmetries", which is equivalent to the term "coding asymmetries". Originally, the motivation to study such overt-zero oppositions came from structuralism, where binary markedness contrasts and analyses involving zero signs were prominent (Jakobson, 1939). Greenberg (1966) discussed such contrasts in terms of "markedness", though he also noted the strong correlation with frequency of use and even proposed that frequency of use could be the explanatory factor. Since the 1970s, syntactic typology has been an active field where many new universals have come to the fore. Coding asymmetries can be found, for instance, in the oppositions of present tense to future tense, singular to plural, and third person to first person: the former concepts are more frequent and tend to be shorter than the latter (cf. Greenberg, 1966, Croft, 2003; Haspelmath, 2008b). There is, however, an important constraint: coding asymmetries are a crosslinguistic feature of languages on all continents, but not every language exhibits those asymmetries. For instance, Siewierska (2005) carried out a worldwide study of third-person markers on verbs, which found that in a majority of languages zero coding cannot be found, and that thus there is no coding asymmetry. Coding asymmetries, on the other hand, are a language universal, requiring a functional explanation of this phenomenon.

Haspelmath (2008) advocates predictability as a functional explanation, since for instance presenttense, singular and third-person singular verb forms are more frequent than future, plural and first-person singular forms, respectively. In order to capture this phenomenon, and in line with Zipfian law, Haspelmath et al. (2014) formulate the Form-Frequency-Correspondence principle (FFC), which encapsulates the tendency of languages to use less coding material for more frequent expressions. When two grammatical patterns that differ minimally in meaning (i.e. patterns that form a semantic opposition) occur with significantly different frequencies, the less frequent pattern tends to be overtly coded (or carry more coding material), while the more frequent pattern tends to be zero-coded (or carry less coding material) (Haspelmath et al., 2014). ${ }^{2}$

As a language universal, the grammatical relationships described above should show in any language, and there is, for instance, massive evidence from empirical studies (Greenberg, 1966; Bybee, 1985;

\footnotetext{
${ }^{2}$ Significance can be checked by t- or chi-square-tests, which determine whether grammatical patterns come from the same distributions (t-tests) or whether the actual frequency distribution of grammatical pattern deviates from the expected distribution (chi-square-test).
} 
Croft, 1990; Bybee et al., 1994; Haspelmath, 1998) that the frequency of use of the forms mentioned above should be universally skewed. Haspelmath and Karjus (2016) provide evidence for frequency differences with number marking asymmetries in five languages. However, verbal aspect has rarely been a topic of research on coding asymmetries and involves research on the interaction between coding, information and length.

The overall picture is somewhat unclear: Bybee (1985) found no strong evidence that the perfective or imperfective aspects are overtly coded. Russian is a different case: Fenk-Oczlon (1990) found that some verb types tend to be basic imperfectives, namely verb types that use the imperfective, and others tend to be basic perfectives - those verb types that use the perfective aspect more frequently. Bickerton (1981) observed a systemic distribution of overt- and zero-coded forms in many creole languages with actional and aspect classes: when telic dynamic verbs are not overtly marked, they have perfective (and generally past-tense) meaning. Stative verbs have imperfective (and generally present-tense) meaning when they are used without an overt marker. As a default, telic verbs tend to occur in the perfective aspect, and atelic verbs tend to occur in the imperfective aspect. It is thus a cross-linguistic phenomenon that canonical (i.e. more frequent) combinations tend to be overtly zero-coded. In contrast, combinations that occur more rarely, namely non-canonical combinations, tend to be overtly coded by special imperfective or perfective markers, respectively, which are limited in application, as illustrated for Mandarin Chinese in Table 1:

Table 1. Coding asymmetries in Mandarin Chinese.

\begin{tabular}{llll}
\hline & & actional classes & \\
\cline { 3 - 3 } & & atelic & telic \\
\hline aspect classes & imperfective & chì-fàn & guà zhe \\
& 'eat' & 'is hanging' \\
\cline { 2 - 3 } & perfective & tā chī-fàn le & guà \\
& 'he ate' & 'hang' \\
\hline
\end{tabular}

The telic verb guà 'hang' occurs unmarked with the perfective aspect, which is to say, in a default form. However, with the imperfective aspect, the telic is marked by means of the particle zhe which follows it. The opposite situation arises with the atelic verb chi-fàn 'eat': with the imperfective aspect, this verb is not marked and thus has a default form. But with the unexpected perfect aspect, this verb is marked by the particle 'le' which follows it and has a non-default-form. If a default form is encountered, this is the expected, unsurprising case. The non-default form, on the other hand, represents the unexpected, surprising case.

In terms of information theory, coding economy embodies the fact that expectable forms have a shorter length than unexpectable ones. Although FFC describes this tendency, it does not consider the complex interaction of coding/character length, frequency and entropy, but only the relationship between form and frequency. The complex relationship between length, frequency and entropy which we hypothesize to hold in natural languages leads to the research question: are FREQUENCY and the entropy measure AVERAGE INFORMATION CONTENT predictors of length of aspect-marked verbs and how high is their respective influence? Following Piantadosi, Tily and Gibson (2011), we interpret FREQUENCY as the classical Shannon Information Content (see Section 3), and, in addition, use the entropy measure AVERAGE INFORMATION CONTENT (henceforth IC) as a predictor. IC can intuitively be interpreted as the average amount of information that a word conveys in all its contexts in a large corpus. In addition, we investigate the influence of the DEFAULT effect on length. In Table 1, an example of default- and non-default coding has been given: the DEFAULT feature (Celano et al., 2018) refers to markedness of words. Expected forms tend to be shorter and can be classified as default; unexpected forms tend to be longer and marked, and are non-default. Expectability results from frequencies and thus probabilities: the probability of the occurrence of default forms is higher than that of non-default forms. Markedness had been described as "coding asymmetries" (see Table 1). Consider an additional example from Inuktitut (Bohnemeyer and Swift, 2004), given in Table 2. The telic verb ani 'go out' is default-coded when it occurs in the expected perfective aspect. But when this verb occurs with imperfective aspect, it is non-default coded. Note that the default form is shorter than the nondefault form. 
Table 2. Default and Non-Default coding of telic verb ani 'go out' in Inuktitut.

\begin{tabular}{lll}
\hline aspect & example & coding \\
Imperfective & ani-liq 'is going out' & NON-DEFAULT \\
Perfective & ani 'went out' & DEFAULT \\
\hline
\end{tabular}

Non-default-coding causes, in the framework of information and surprisal theory, a surprisal-effect. Hale (2001) in surprisal theory and Levy (2008) define surprisal as the negative logarithm of the (conditional) probability of a word in a context, but context can be determined in various ways, and even be extrasentential.

The research question is in particular based on the findings of Piantadosi et al. (2011; see Section 2) and Levshina (2017; see Section 2), who both claim that IC is the strongest predictor of word length in general, employing unstructured co-occurrences of verbs (Piantadosi et al., 2011) and verb dependents (Levshina 2017). Following Celano et al. (2018), we estimate IC from verb dependents, but unlike Piantadosi (2011), we use IC to predict lengths of aspectual verb forms. This is motivated by a previous study on Russian (Celano et al., 2018). In that study, IC was estimated from syntactic dependency labels and significant impact of IC on verb length was observed. The present study aims to test whether IC from syntactic dependency-label contexts has an impact in a greater number of languages.

Just as Piantadosi et al. (2011) do, we use the number of characters the words have as values for the dependent variable, i.e. their orthographic length, since this information is available in the corpora. This aligns with Piantadosi et al. (2011) in viewing orthographic length as highly correlated with phonetic length.

The FFC principle (Haspelmath et al., 2014, see above) suggests that if there is a statistically significant frequency difference between imperfective and perfective word forms for a given lemma, the verb forms associated with the most frequent aspect category, i.e. those showing default aspect, should (on average) be shorter than those having non-default aspect. 'Imperfective' and 'perfective' are sub-concepts of aspect. Aspect defines the (temporal) perspective taken on an event (see 2012) and a distinction is commonly made between lexical and grammatical aspect (Sasse, 2006; Velupillai, 2012), even if their semantics is different (Johanson, 2000). An interaction between both aspect types is the correlation between telicity and perfectivity. Telicity describes whether or not a verb/predicate has an inherent goal or endpoint. Telic verbs/predicates have an inherent goal or endpoint, whereas atelic verbs/predicates do not. Regarding (im)perfectivity, the perspective of the perfective aspect is on the whole event, while that of the imperfective aspect lies within the event (Velupillai, 2012).

\section{Related work}

Predictability, prominently of phonetic duration, has been approached utilizing short-distance measures of probability and entropy, for instance by determining mutual information, joint probability, conditional probability (Bybee and Scheibman, 1999; Gregory et al., 1999; Aylett and Turk, 2004; Pluymaekers et al., 2005) and long-distance measures (Gahl and Garnsey, 2004). The latter demonstrated that a bias towards a particular syntactic subcategorization affects pronunciation length. Studies by Fowler and Housum (1987), Fowler (1988), Hawkins and Warren (1994) and Bard et al. (2000) revealed that occurrence of words in a previous context affects the accentuation of those words. A study by Bell, Brenier, Gregory, Girand and Jurafsky. (2009) provides evidence that in conversational English, conditional probability and word frequency are the strongest predictive factors for the duration of content words.

The entropy measure IC used in my model is estimated from conditional probabilities (the estimate is given in Section 3 below) and is a variant of conditional entropy. Conditional probabilities as input of classifiers for dependency parsing tasks were used by Sagae and Tsujii (2007) (probabilistic Left-RightAlgorithm parser) and by Li, Cheng, Liu and Keller (2018) (Transition-based parser).

Demberg et al. (2013) argue that models based on conditional probabilities, for instance in the framework of surprisal theory (see above, Hale, 2001), have cognitive plausibility since humans tend to make predictions from contexts when they parse natural language (cf. Altmann and Kamide, 1999). Hale (2001), Levy and Jaeger (2007), Levy (2008) and Jaeger (2010) use the concept of surprisal to describe the effect of conditional probabilities of words on (human) sentence processors. Surprisal is a concept from information theory and, as pointed out above, corresponds to IC. Piantadosi et al. (2011) 
investigate the correlation between IC and word length: it emerges for 10 Indo-European languages that IC, calculated on the basis of syntactic context (bigrams, trigrams, and four-grams), is a better predictor of word length than frequency. According to them, the effect of frequency is largely due to its correlation with information content. They ascribe the established correlation of word length and information content to the principle of uniform information density, which briefly stated holds that the information rate of communication over time is kept as constant as possible (Piantadosi et al., 2011).

More recently, Levshina (2017) has investigated whether the length of words can be predicted better if the IC score is calculated based on syntactic dependents rather than on co-occurrence frequencies (ngrams), as in Piantadosi et al. (2011). Her study confirms the hypothesis that words with higher IC tend to be longer in most languages.

\section{Data and method}

The data resource is UD treebanks (v. 2.1), which currently represent the largest annotated and typologically diverse corpus, comprising 102 treebanks and 60 languages. We employed a mixedeffects model (Bates et al., 2015) and the underlying assumption was that, if one could identify a default aspect for each verb lemma, i.e. the aspect a verb is primarily associated with on the basis of its lexical value, then one could try to test whether average word lengths of imperfective and perfective word forms can be predicted by the fixed effects DEFAULT, FREQUENCY and IC respectively, while controlling for lemma as a random effect. ${ }^{3}$ A successful application of that model to all the UD treebanks where aspect is encoded as a verb morphological feature would provide evidence for the existence of an aspect-related cōing asymmetry.

In the UDD corpus, only 45 treebanks contain annotation for the morphological feature aspect. We excluded 12 of these treebanks: all Czech treebanks, UD_Gothic, UD_Sanskrit, UD_Hungarian and UD_North_Sami have different lemmas for imperfective and perfective verb forms and it is therefore impossible to compare their word-length differences while keeping the lemma as a constant; on the other hand, UD_Arabic-NYUAD does not contain word forms for copyright reasons, while the UD_Chinese treebanks (UD_Chinese and UD_Chinese-PUD) have aspect encoded but not on verbs. Ramm et al. (2017) present a tool for automatic annotation of, among other things, aspectual information for English, French and German. These languages, however, are not in the focus of the present study since overt aspect coding in them is sparse.

In order to render the data cross-linguistically uniform, aspect oppositions within each treebank are reduced to the binary imperfective-perfective distinction, which can be found in all languages displaying morphological aspect. We subsumed the habitual and progressive aspects under the imperfective aspect, and the resultative aspect under the perfective aspect. Verb forms in the prospective aspect have been ignored, since its value is not clear with respect to the imperfective and perfective opposition. ${ }^{4}$ This aspect reduction concerns the following treebanks (see Table 3):

\footnotetext{
${ }^{3}$ A reviewer suggested that length of the lemma should not be treated as a random effect. Instead, the predicted variable should be the length of the verb form relative to the lemma length. In our understanding of linear mixed models, this is precisely what the linear model in this study does: it predicts the length of a verb form relative to the length of the respective. The effect 'lemma' is a covariate and a characteristic feature of mixed models is that they can control covariates. By adding covariates, it is possible to improve significantly the accuracy of the model and this has a significant impact on the final results of the analysis. Embedding a covariate in the model can reduce the error in the model and increase the selectivity of factor testing.

4 It can also be argued that the prospective as defined in UD (http://universaldependencies.org $/ \mathrm{u} /$ feat/Aspect.html) is not really a type of aspect, in that its definition centres around relative time in reference to another action.
} 
Table 3. Treebanks with aspect reduction.

\begin{tabular}{ll}
\hline UD Treebanks & original aspect categories \\
UD_Basque & Perf, Imp, Prog, Prosp \\
UD_Buryat & Imp, Hab, Perf, Prog \\
UD_Hindi-PUD & Perf, Imp, Prog \\
UD_Kurmanji & Prog, Perf) \\
UD_Marathi & Imp, Perf, Prosp, Hab \\
UD_Old_Church_Slavonic & Perf, Res, Imp \\
UD_Turkish-PUD & Perf, Hab, Prog, Imp, Prosp \\
UD_Slovak & Perf, Imp, Imp,Perf \\
UD_Tarkish & Perf, Prog, Imp, Rapid, DurPerf, \\
& ProgRapid \\
\hline
\end{tabular}

We counted all the imperfective and perfective word forms of each lemma and used Pearson's $\chi^{2}$ tests to determine whether differences between the two aspect categories of a lemma are statistically significant $(\mathrm{p}<.05)$ : if so, we take the most frequent aspect category as the default for a given lemma and calculate the (character) word length of the imperfective and perfective aspects as the average of the lengths of all the word forms within each aspect category. The languages with significant differences between perfective and imperfective word forms are listed in Table $4 .^{5}$

Table 4. UD treebanks with the number of lemmas for which the frequency difference between perfective and imperfective word forms is statistically significant $(\mathrm{p}<0.05)$.

\begin{tabular}{ll}
\hline UD Treebanks & Lemma \\
UD_Ancient_Greek & 42 \\
UD_Ancient_Greek-PROIEL & 205 \\
UD_Arabic & 181 \\
UD_Arabic-PUD & 3 \\
UD_Basque & 82 \\
UD_Bulgarian & 6 \\
UD_Greek & 58 \\
UD_Hindi & 62 \\
UD_Latin & 9 \\
UD_Latin-ITTB & 68 \\
UD_Latin_PROIEL & 164 \\
UD_Latvian & 6 \\
UD_Marathi & 6 \\
UD_Old_Church_Slavonic & 18 \\
UD_Polish & 4 \\
UD_Russian-SynTagRus & 571 \\
UD_Slovenian & 3 \\
UD_Urdu & 25 \\
\hline
\end{tabular}

\footnotetext{
${ }^{5}$ A reviewer remarked that statistical differences in the counts of perfective vs. imperfective instances of a verb are not a good criterion for identifying the default aspect. In Slavic languages, for instance, a single verb usually would have a single aspect, e.g. a perfective verb is always perfective, be this its default aspect or not. In Universal Dependency Treebanks, that is the data resources in this study, the aspect of each verb form is specified. For example, the Russian lemma уметь 'able to be' occurs in total with 124 word forms in the corpus 'ru_syntagrusud-train.conllu', 58 times it occurs with imperfect aspect and 66 times with perfective aspect in this corpus. Only lemmas were used for the study in which the difference between the perfective and imperfective occurrences is statistically significant. The more frequent aspect forms were taken as default. Thus, verbs that occur exclusively in one aspect form were not included in the study. In follow-up studies, we are currently experimenting with other methods of distinguishing between default and non-default forms, for example by normalized thresholds between perfective and imperfective occurrences of verbs.
} 
The average word-form lengths for each lemma are used because a direct comparison of lengths of verb word forms agreeing in any morphological feature other than aspect is not always possible, since it is not always the case that all imperfective word forms in a treebank are also confirmed as perfective (and vice versa). Since word length can to a great extent be affected by non-aspect-related morphemes (such as 'person' or 'number', which can change in verb conjugation), average length allows us to maximize the number of verb lemmas available for comparison.

In the mixed-effect model, we took FREQUENCY as Shannon Information Content (Shannon and Weaver, 1948), which is to say, the negative logarithm of the probability of a verb form $v$.

(1) $I C_{\text {SHANNON }}=-\log (p(v))$

We controlled for LEMMA by defining it as a random effect in the model since the absolute difference between lengths depends on the specific lemma. The predictors were negatively tested for collinearity. This indicates that DEFAULT is not a masked FREQUENCY predictor. The estimation of IC is given in (2) (Cohen Priva, 2008; Piantadosi et al., 2011):

(2) IC $=-\frac{1}{N} \sum_{i=1}^{N} \log \left(p\left(v \mid c_{i}\right)\right)$

IC is the negative log conditional probability of a verb form $v$ (marked as imperfective or perfective aspect) given the context $c_{i}$, which is defined syntactically as the set of the syntactic labels of the direct dependents of $v$ : 'advmod', 'advcl', 'obl', 'nsubj', 'nsub:pass', and 'csubj:pass'.

nsubj. Examples of the labels are given in Table 5:

Table 5. Syntactic labels of the direct dependents of $v$ and linguistic examples.

\begin{tabular}{|c|c|c|c|}
\hline label & & dependency relation & example \\
\hline advmod & & adverbial modifier & that is very delicious \\
\hline advcl & & adverbial clause modifier & $\begin{array}{l}\text { they played when the moon } \\
\text { was shining }\end{array}$ \\
\hline & - & oblique nominal & run with pleasure \\
\hline nsubj & & nominal subject & they laughed \\
\hline nsubj:pass & & passive nominal subject & the gold was found \\
\hline csubj:pass & & clausal passive subject & $\begin{array}{l}\text { it cannot be predicted when it } \\
\text { will happen }\end{array}$ \\
\hline
\end{tabular}

We restrict IC to only the preceding labels because, on a theoretical basis, we expect them to be the most representative ones for each $v$ (conjuncts and dependents broadly definable as function words were excluded, as they are notoriously not verb-specific). As UD syntactic annotation is not meant to (exactly) capture verb argument structure (e.g. oblique dependents can correspond to either adjuncts or arguments), we include the labels 'advmod', 'advcl', and 'obl' in my IC definition: in the pilot study on UD_Russian-SynTagRus (Celano et al., 2018), this selection provided the best results with the mixed-effects model, and we therefore stick to it. The summation is calculated on the basis of the occurrences of $v$, and the result divided by $\mathrm{N}$, i.e. the total frequency of contexts $v$ occurs in. Once default aspects and their frequency and IC have been calculated for the lemmas in each treebank (both frequency and IC being calculated as negative logarithms), we fit a mixed-effects model in (3) with a random effect for lemma for each treebank:

(3) $y_{j}=\beta_{0}+\beta_{1}$ Def $_{j}+\beta_{2}$ Freq $_{j}+\beta_{3} I C_{j}+\beta_{4}\left(\right.$ Freq $\left._{j}: I C_{j}\right)+W_{j}+\varepsilon_{j}$

In (3), $y$-values are the average word-form lengths of verb lemmas. Predictors are DEFAULT, FREQUENCY (see formula 1), IC (see formula 2), and the interaction between FREQUENCY and IC. The model also contains a by-lemma adjustment to the intercept $(W)$, which represents the random effect and an error term $\varepsilon$. As the length of a verb word form is significantly affected by its base 
form/root, which in turn affects lemma length, adding a random effect for lemma is expected to be a rational improvement of the model.

\section{Results}

A significant relationship between aspect word length and DEFAULT came to light in only 6 of the 18 treebanks (see Table 6):

Table 6. Treebanks with significant relationship between aspect word length and DEFAULT.

\begin{tabular}{ll}
\hline treebank & p of predictor DEFAULT \\
UD_Ancient Greek-PROIEL & $<.001$ \\
UD_Basque & $<.001$ \\
UD_Hindi & $<.001$ \\
UD_Marathi & $<.05$ \\
UD_Polish & $<.001$ \\
UD_Russian-SynTagRus & $<.001$ \\
\hline
\end{tabular}

Four treebanks turn out to have a significant relationship only between aspect word length and FREQUENCY (or the interaction between FREQUENCY and IC) (see Table 7):

Table 7. Treebanks with a significant relationship between aspect word length and FREQUENCY.

\begin{tabular}{ll}
\hline treebank & p of predictor FREQUENCY \\
UD_Greek & $<.001$ \\
UD_Latin-ITTB & $<.01$ \\
UD_Latin-PROIEL & $<.05$ \\
UD_Urdu & $<.05$ \\
\hline
\end{tabular}

Two treebanks have a significant relationship between not only aspect word length and FREQUENCY but also aspect word length and IC, as Table 8 shows:

Table 8. Treebanks with a significant relationship between aspect word length, FREQUENCY and IC.

\begin{tabular}{ll}
\hline treebank & p of predictors FREQUENCY and IC \\
UD_Ancient_Greek & $<.05<.05$ \\
UD_Old_Church_Slavonic & $<.001<.01$ \\
\hline
\end{tabular}

Only in UD_Basque, UD_Hindi, UD_Marathi and UD_Russian-SynTagRus does a positive impact of the predictor DEFAULT emerge - that is to say, default code verbs are longer than non-default-coded verbs (see Figures 2, 3, 4, 6). For UD_Ancient_Greek-PROIEL and UD Polish, we observed a significant but negative correlation between aspect word length and DEFAULT: this means that in these languages, non-default values are associated with, on average, shorter word lengths (see Figures 1 and 3). A significant relationship between FREQUENCY and aspect word length is confirmed in 11 languages. UD Marathi, however, provides counter evidence (see Figure 4).

An interaction between IC and FREQUENCY comes to light in two languages in particular, UD_Ancient_Greek-PROIEL and UD_Russian_SynTagRus (Figure 1 and Figure 6), confirming the results of a previous study (Celano et al., 2018) which focused on the Russian language: the effect of FREQUENCY weakens as IC increases. With uninformative verb forms, the impact of FREQUENCY is high, and vice versa when IC is at its maximum, with the effect of FREQUENCY almost annihilated. The results indicate that IC and FREQUENCY do not have a simultaneous positive impact on the length of verb forms: FREQUENCY reveals its effect when verb forms tend to be less informative, but when they are informative, the relevance of FREQUENCY decreases. This tendency could be expressed in logical terms by saying that either IC has an impact on average lengths or FREQUENCY has a massive impact, but not both at the same time. In the remaining languages the interaction between IC and FREQUENCY is weak or even non-existent, as Figures 2, 3 and 5 exemplify. 

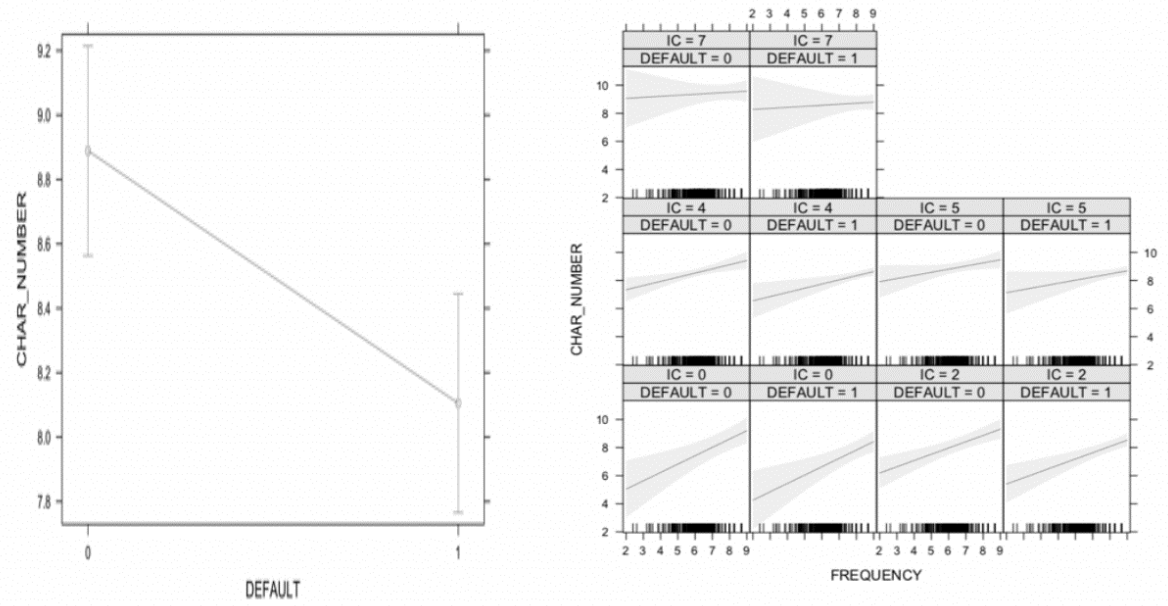

Figure 1. Character number as a function of DEFAULT (left) and mixed-effects fit for function of DEFAULT (right) for UD_Ancient_Greek_PROEIL.
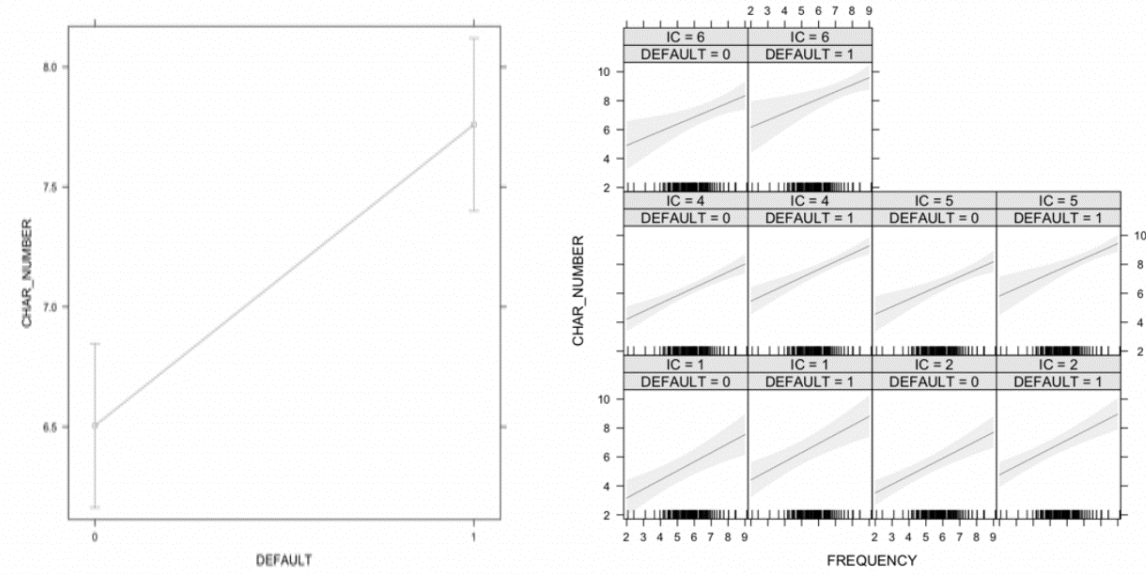

Figure 2. Character number as a function of DEFAULT (left) and mixed-effects fit for function of DEFAULT (right) for UD_Basque.
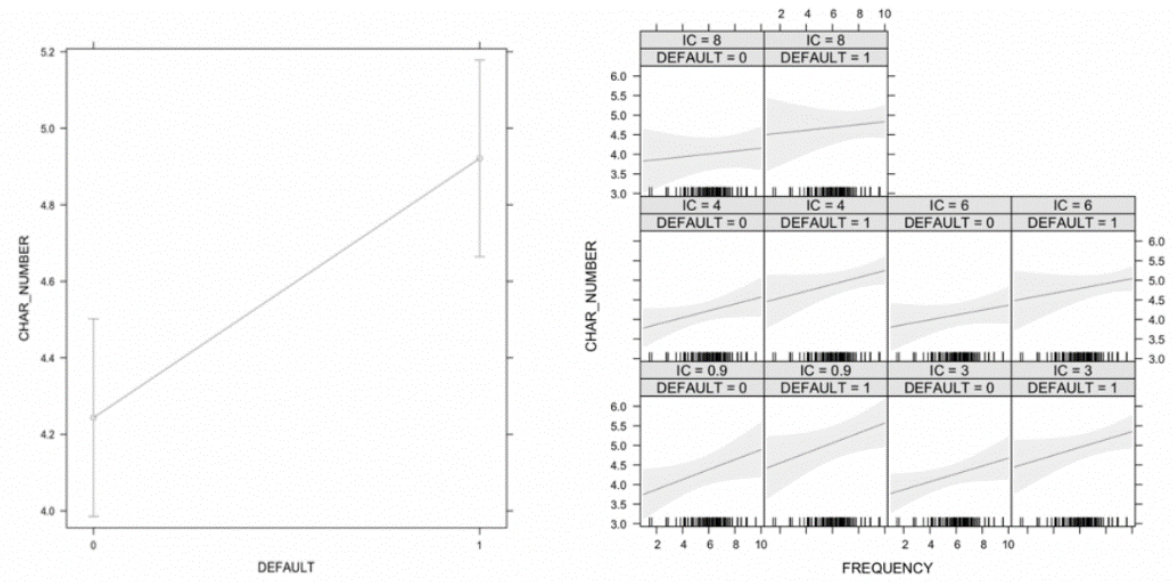

Figure 3. Character number as a function of DEFAULT (left) and mixed-effects fit for function of DEFAULT (right) for UD_Hindi. 


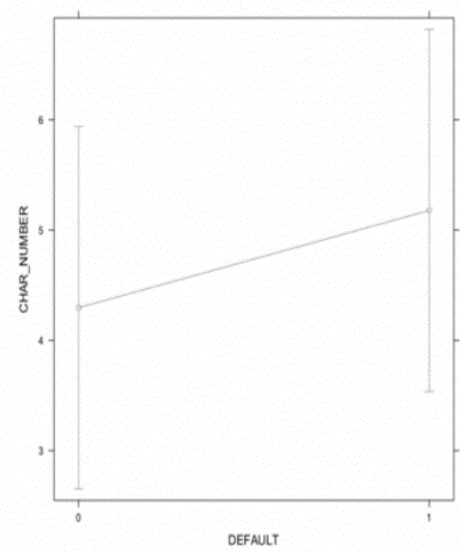

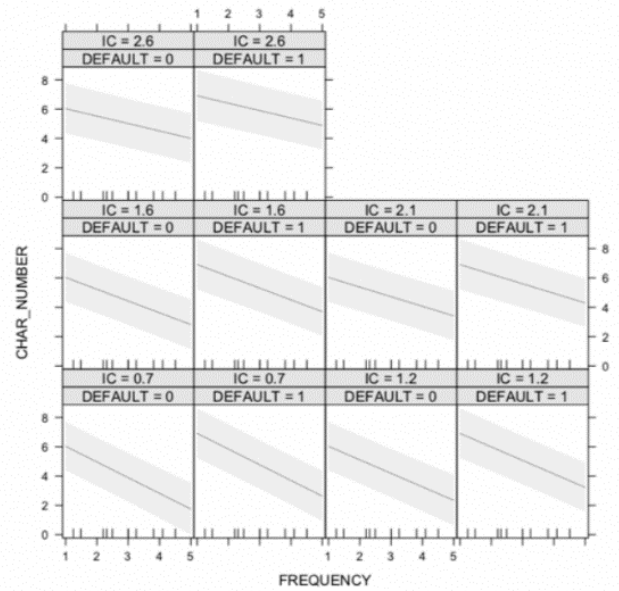

FREQUENCY

Figure 4. Character number as a function of DEFAULT (left) and mixed-effects fit for function of DEFAULT (right) for UD_Marathi.
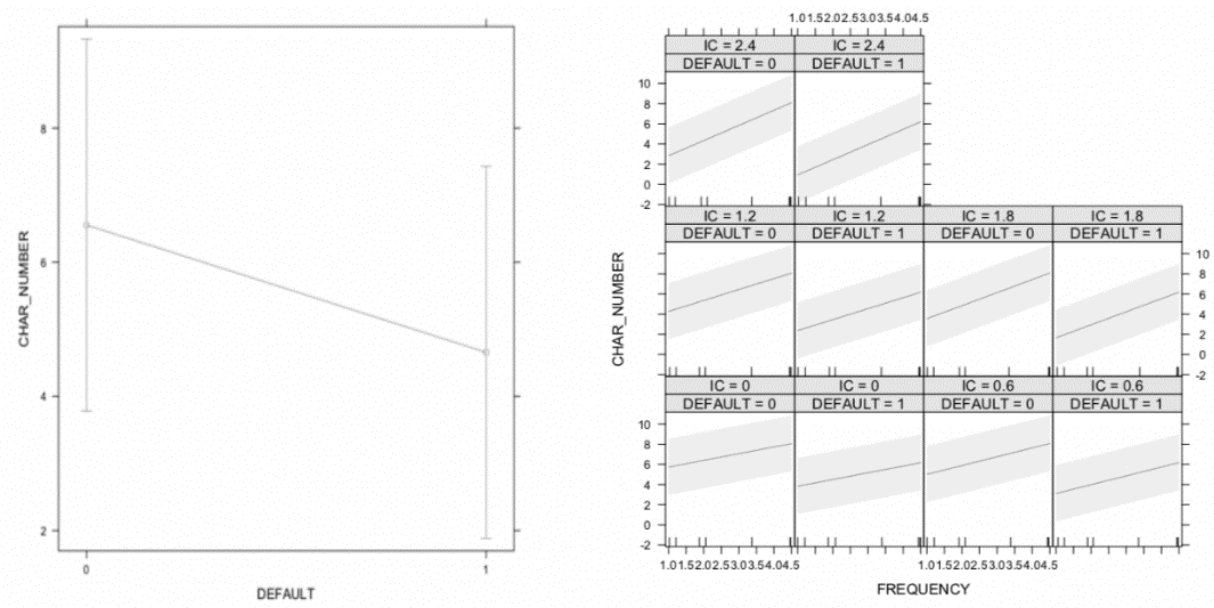

Figure 5. Character number as a function of DEFAULT (left) and mixed-effects fit for function of DEFAULT (right) for UD_Polish.
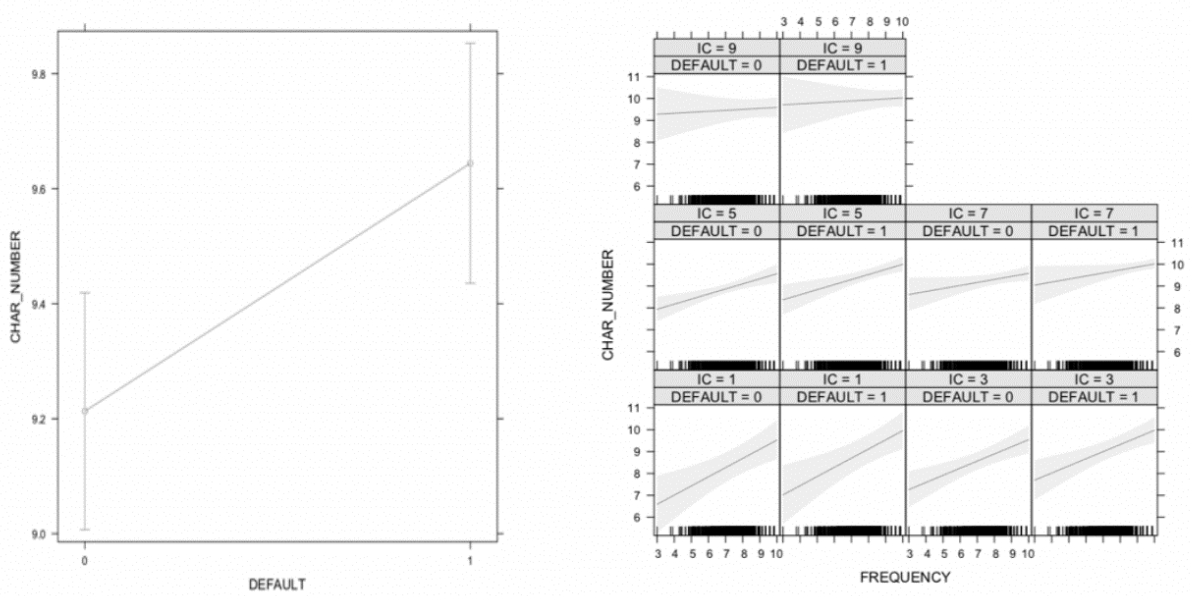

Figure 6. Character number as a function of DEFAULT (left) and mixed-effects fit for function of DEFAULT (right) for UD_Russian_SynTagRus. 


\section{Conclusion}

The results from the mixed-effects model are rather poor, as far as language universals are concerned. Six of the 18 treebanks (UD Latin, UD Bulgarian, UD Slovenian, UD Arabic, UD Arabic-PUD and UD Latvian) do not show any significant relationship between aspect word length and any predictor. We hypothesized that DEFAULT is a predictor of word length, but only 4 treebanks provide evidence for such a relationship and in just 11 out of 18 treebanks is a positive correlation between FREQUENCY and verb length apparent. IC interacting with FREQUENCY reveals its effect on the lengths of verbs in only 2 treebanks.

Based on evidence from more than 900 languages (Bentz and Ferreri Cancho, 2016), the validity of Zipf's law was the point of departure of this study, but the fact that it does not apply in the present study to a couple of languages could be taken as an indication of an overall lack of relationship between average aspect word length and default value. Indeed, coding asymmetries can be interpreted as specifications of Zipf's law, in that a (shorter) default value in a binary linguistic opposition should be expected to occur more frequently than the (longer) non-default one. It should therefore not be surprising that if Zipf's law does not apply overall in the present study, the related coding asymmetry does not either. These preliminary results will be challenged in follow-up studies.

The study brought to light an interaction between FREQUENCY and IC in particular in Ancient Greek and Russian. This could be an indication of linguistic economy: FREQUENCY is just as large as it needs to be in order to predict verb lengths. With highly informative verbs forms, it does not matter whether they are frequent or not, since the effect of the predictor IC suffices and vice versa.

In natural languages, a great variety of principles cooperate and compete to determine language form, and it is reasonable to expect none of them to be valid in all languages. In language universals, research principles are sought which are expected to hold for a large sample of languages rather than for all languages. Similarly, in coding asymmetry research, a hypothesis about a specific coding asymmetry can prove false in a couple of languages without the general hypothesis about coding asymmetries being proved so. More corpus-based research on different coding asymmetries is needed before a stance can be taken; and in addition, as said above, modifications to the present study are needed too, addressing the following questions: (1) Will exploiting larger corpora lead to better results? (2) How can default aspect value for lemmas can alternatively be identified? (3) Will other contexts for the estimation of information content yield better results? and (4) Should average verbs lengths be replaced by the individual lengths of verb forms?

\section{Acknowledgements}

This work was funded by the Deutsche Forschungsgemeinschaft (DFG, German Research Foundation) - project number: 357550571.

\section{References}

Altmann, G. T., M., Kamide, Y., 1999. Incremental interpretation at verbs: Restricting the domain of subsequent reference. Cognition, vol. 73, no. 3, pp. 247-264.

Aylett, M.,Turk, A., 2004. The Smooth Signal Redundancy Hypothesis: An explanation for relationships between redundancy, prosodic prominence, and duration in spontaneous speech. Language and Speech, vol. 47, no.1, pp. $31-56$.

Bard, E. G., Anderson, A., H, Sotillo, C., Aylett, M., Doherty-Sneddon, G. and Newlands, A., 2000. Controlling the intelligibility of referring expressions in dialogue. Journal of Memory and Language, vol. 42, pp. $1-22$.

Bates, D., Mächler, M., Bolker, B. and Walker, S., 2015. Fitting Linear Mixed-Effects Models using lme4. Journal of Statistics Software, vol. 67, no. 1, pp.- 1-48.

Bell, A, Brenier, J.M., Gregory, M. L., Girand, C. and Jurafsky, D., 2009. Predictability effects on durations of content and function words in conversational English. Journal of Memory and Language, vol. 60, pp. $92-111$.

Bentz, C., Ferreri Cancho, R., 2016. Zipfs law of abbreviation as a language universal. Paper presented at The Leiden Workshop on Capturing Phylogenetic Algorithms for Linguistics. Leiden, The Netherlands.

Bickerton, D., 1981. Roots of language. Ann Arbor: Karoma. 
Bohnemeyer, J., Swift, M., 2004. Event realization and default aspect. Linguistics and Philosophy, vol. 27 , no. 3, pp. $263-296$.

Bybee, J. L., Scheibman. J., 1999. The effect of usage on degrees of constituency: the reduction of don't in English. Linguistics, vol. 37, no. 4, pp. 575 - 596.

Bybee, J. L., 1985. Morphology: A Study of the Relation between Meaning and Form. Amsterdam: Benjamins.

Bybee, J. L., 1994. The grammaticization of zero: asymmetries in tense and aspect systems. In: W. Pagliuca, ed. Perspectives on grammaticalization, vol. 109. Amsterdam: Benjamins, pp. 235 - 254.

Celano, G. G. A., Richter, M., Voll, R. and Heyer, G., 2018. Aspect coding asymmetries of verbs: The case of Russian. In: A. Barbaresi, H. Biber, F. Neubarth, R. Osswald. (eds.) KONVENS 2018. PROCEEDINGS of the 14th Conference on Natural Language Processing, pp. 34 - 39

Cohen Priva, U., 2008. Using information content to predict phone deletion. Paper presented at The 27th West Coast Conference on Formal Linguistics. University of California, Los Angeles, May 16 - 18, 2008.

Croft, W., 2003. Typology and universals. 2nd edition. Cambridge University Press.

Croft, W., 2012. Verbs: Aspect and causal structure. Oxford University Press.

Demberg, V., Keller, F. and Koller. A., 2013. Incremental, predictive parsing with psycholinguistically motivated tree-adjoining grammar. Computational Linguistics, vol. 39, no. 4, pp. 1025-1066.

Fenk-Oczlon, G., 1990. Ikonismus versus Ökonomieprinzip: Am Beispiel russischer Aspekt- und Kasusbildungen. Papiere zur Linguistik, vol. 42, no. 1, pp. 49 - 69.

Fowler, C. A., 1988. Differential shortening of repeated content words produced in various communicative contexts. Language and Speech, vol. 31, pp. $307-319$.

Fowler, C. A., Housum. J., 1987. Talkers' signaling of "new" and "old" words in speech and listeners' perception and use of the distinction. Journal of Memory and Language, vol. 26, pp. 489 504.

Greenberg, J. H., 1963. Some universals of grammar with particular reference to the order of meaningful elements. In: J. H. Greenberg, ed. Universals of language. Cambridge. MA: MIT Press, pp. 73 - 113.

Greenberg, J.H., 1966. Language universals, with special reference to feature hierarchies. The Hague: Mouton.

Gregory, M. L., Raymond, W. D., Bell, A., Fosler-Lussier, E. and Jurafsky, D., 1999. The effects of collocational strength and contextual predictability in lexical production. Chicago Linguistics Society (CLS-99), vol. 35, pp. $151-166$.

Hale. J., 2001. A probabilistic Earley parser as a psycholinguistic model. Paper presented at NAACL. Carnegie Mellon University, Pittsburgh, 2-7 June 2001.

Haspelmath, M., 2008. Creating economical patterns in language change. In: J. Good, ed. Linguistic universals and language change. Oxford: Oxford University Press, pp. 185-214.

Haspelmath, M., Calude, A., Spagnol, M., Narrog, H. and Bamyacì, E., 2014. Coding causal-noncausal verb alternations: A form-frequency correspondence explanation. Journal of Linguistics, vol. 50, no. 3, pp. 587-625.

Haspelmath, M., Karjus, A., 2017. Explaining asymmetries in number marking: Singulatives, pluratives and usage frequency. Linguistics, vol. 55, no. 6, pp. 1213-1235.

Hawkins, S., Warren, P., 1994. Implications for lexical access of phonetic influences on the intelligibility of conversational speech. Journal of Phonetics, vol. 22, pp. $493-511$.

Jaeger, T. F. 2010. Redundancy and reduction: Speakers manage information density. Cognitive Psychology, vol. 61, no 1. pp. 23-62.

Jakobson, R., 1939. Signe zéro. Mélanges linguistiques offerts à Charles Bally, pp. 143 - 152. Genève.

Johanson, L., 2000. Viewpoint operators in European languages. In: Ö. Dahl, ed. Tense and Aspect in the Languages of Europe. Berlin: Mouton de Gruyter, pp. 27-187.

Levshina, N., 2017. Communicative efficiency and syntactic predictability: A crosslinguistic study based on the Universal Dependencies corpora. Paper presented at The NoDaLiDa 2017 Workshop on Universal Dependencies (UDW 2017). Gothenburg, Sweden, May 22 - 24, 2017.

Levy, R., Jaeger, T. F., 2007. Speaker Optimize Information Density Through Syntactic Reduction. Proceedings of the 20th Conference on Neural Information Processing Systems (NIPS).

Levy, R. 2008. Expectation-based syntactic comprehension. Cognition, vol. 106, no. 3, pp. 1126 -77. 
Levy, R. 2013. Memory and Surprisal in Human Sentence Comprehension. In: R. van Gompel, ed. Sentence Processing. Hove: Psychology Press, pp. 78-114.

Li, B., Cheng, J., Liu, Y. and Keller, F., 2018. Dependency Grammar Induction with a Neural Variational Transition-based Parser. Available at https://arxiv.org/abs/1811.05889

Nivre, J., Agic' Ž., Ahrenberg, L. et al., 2017. Universal Dependencies 2.0-CoNLL 2017 Shared Task Development and Test Data, LIN- DAT/CLARIN digital library at the Institute of Formal and Applied Linguistics (ÃŽFAL), Faculty of Mathematics and Physics, Charles University, Prague.

Piantadosi, S.T., Tily, H. and Gibson, E., 2011. Word lengths are optimized for efficient communication. PNAS, vol. 108, no. 9, pp. 3526-3529.

Pluymaekers, M., Ernestus, M. and Baayen, H., 2005. Articulatory planning is continuous and sensitive to informational redundancy. Phonetica, vol. 62, no. 2-4, pp. 146-159.

Ramm, A., Loáiciga, S., Friedrich, A. and Fraser, A., 2017. Annotating tense, mood and voice for English, French and German. Paper presented at The 55th Annual Meeting of the Association for Computational Linguistics, demo session (ACL). Vancouver, Canada, July 3-August 2, 2017.

Sagae. K., Tsujii, J., 2007. Dependency Parsing and Domain Adaptation with LR Models and Parser Ensembles. Proceedings of the CoNLL 2007 Shared Task in the Joint Conferences on Empirical Methods in Natural Language Processing and Computational Natural Language Learning (EMNLPCoNLL'07 shared task), pp. 1044-1050. Prague, Czech Republic.

Sasse, H.-J., 2006. Aspect and Aktionsart. In: E. K. Brown, ed. Encyclopedia of language and linguistics. Boston: Elsevier, pp. 535-538.

Shannon, C., E., Weaver, W.,1948. A mathematical Theory of Communication. The Bell System Technical Journal, vol. 27, 379-423, pp. 623-656.

Velupillai, V., 2012. Zero coding in tense-aspect systems of creole languages. Amsterdam: John Benjamins.

Zipf, G. K., 1936. The Psychobiology of Language. London: Routledge.

Zipf, G. K., 1949. Human Behavior and the Principle of Least Effort. New York: Addison-Wesley. 